\title{
Selective vulnerability in neurodegeneration: insights from clinical variants of Alzheimer's disease
}

\author{
Niklas Mattsson, ${ }^{1}$ Jonathan M Schott, ${ }^{2}$ John Hardy, ${ }^{3}$ Martin R Turner, ${ }^{4}$ \\ Henrik Zetterberg ${ }^{3,5}$
}

${ }^{1}$ Clinical Memory Research Unit, Faculty of Medicine, Lund University, Lund, Sweden ${ }^{2}$ Dementia Research Centre, UCL Institute of Neurology, London, UK

${ }^{3}$ Department of Molecular Neuroscience, UCL Institute of Neurology, London, UK

${ }^{4}$ Nuffield Department of Clinical Neurosciences, Oxford University, Oxford, UK ${ }^{5} \mathrm{Clinical}$ Neurochemistry Laboratory, Department of Psychiatry and Neurochemistry, Institute of Neuroscience and Physiology, The Sahlgrenska Academy at University of Gothenburg, Mölndal, Sweden

\section{Correspondence to} Dr Henrik Zetterberg, Department of Psychiatry and Neurochemistry, Sahlgrenska University Hospital, Mölndal S-431 80, Sweden; henrik. zetterberg@clinchem.gu.se

Received 8 September 2015 Revised 26 November 2015 Accepted 5 December 2015 Published Online First

8 January 2016

\section{CrossMark}

To cite: Mattsson $\mathrm{N}$, Schott JM, Hardy J, et al. J Neurol Neurosurg Psychiatry 2016;87:1000-1004.

\section{ABSTRACT}

Selective vulnerability in the nervous system refers to the fact that subpopulations of neurons in different brain systems may be more or less prone to abnormal function or death in response to specific types of pathological states or injury. The concept has been used extensively as a potential way of explaining differences in degeneration patterns and the clinical presentation of different neurodegenerative diseases. Yet the increasing complexity of molecular histopathology at the cellular level in neurodegenerative disorders frequently appears at odds with phenotyping based on clinically-directed, macroscopic regional brain involvement. While crossdisease comparisons can provide insights into the differential vulnerability of networks and neuronal populations, we focus here on what is known about selective vulnerability-related factors that might explain the differential phenotypic expressions of the same disease - in this case, typical and atypical forms of Alzheimer's disease. Whereas considerable progress has been made in this area, much is yet to be elucidated; further studies comparing different phenotypic variants aimed at identifying both vulnerability and resilience factors may provide valuable insights into disease pathogenesis, and suggest novel targets for therapy.

\section{INTRODUCTION}

While the amyloid plaque and neurofibrillary $\tau$ tangle pathology of Alzheimer's disease (AD) is often considered synonymous with its commonest clinical manifestation, that is, a progressive amnestic syndrome, individual patients have different constellations and degrees of cognitive symptomatology, with some having sufficiently unusual phenotypes that can be considered as having distinct disease variants. ${ }^{1}$ These include patients with prominent dysexecutive or behavioural problems (sometimes called frontal AD), ${ }^{2}$ patients presenting with word-finding difficulties and pauses in speech (logopenic variant of primary progressive aphasia, lvPPA), ${ }^{3}$ patients with various combinations of cortical visual dysfunction, apraxia and dyscalculia with relative sparing of episodic memory (posterior cortical atrophy, PCA), ${ }^{4}$ and patients with asymmetric rigidity and apraxia, extrapyramidal dysfunction and symptoms related to parietotemporo-occipital cortical involvement (corticobasal syndrome, CBS). ${ }^{5}$ How can the same broad underlying neuropathology be associated with such markedly different clinical manifestations?

\section{Selective vulnerability}

Selective vulnerability in the nervous system refers to the fact that subpopulations of neurons in different brain systems may be more or less prone to abnormal function or cell death in response to specific types of pathological states or injury. The factors underlying this selectivity are well known in some central nervous system diseases, and less well understood or unknown in others. For example, it is well known that certain areas in the brain depend on blood supply from only one or a few arteries due to differences in the circulatory anatomy and are, thus, more vulnerable to an arterial occlusion than other brain areas. ${ }^{6}$ There are also differences in the energy demand of different neurons: hippocampal CA1 neurons and cerebellar Purkinje cells are characterised by particularly high energy consumption and may, therefore, be more vulnerable to hypoxia and other metabolic stressors.'

In neurodegenerative diseases (NDDs), the potential mechanisms underlying selective vulnerability are complex, multifactorial, and incompletely understood. NDDs are characterised by protein misfolding (in the form of intraneuronal inclusions and/or extracellular protein aggregates) and cell death, with the specific protein(s) implicated and associated patterns of neuronal breakdown translating into a clinical phenotype. ${ }^{8}$ Yet the patterns of neuronal breakdown within a clinicallydriven taxonomy may be defined according to various criteria which span several orders of magnitude: from cortical lobes to white matter tracts to soma to proteins to neurotransmitters to genes. Few of these parameters may be studied collectively in vivo, especially presymptomatically. In fact, these have been largely considered in post mortem tissue, that is, at the end of the pathological cascade. This creates a divide between concepts of selective vulnerability at the cellular and cerebral (system) levels.

In NDDs, the toxicity of extracellular protein aggregates (eg, $A \beta$ in senile plaques in $\mathrm{AD}$ ) is most likely distinct from the toxicity of intraneuronal protein inclusions (eg, $\tau$ tangles). Extracellular protein aggregates may injure neurons by leaking diffusible oligomers or proto fibrils of the aggregated protein that may interact with receptors or disrupt membranes in a harmful way, and/or by influencing microglial activation that may in some cases cause deleterious inflammation. In contrast, intraneuronal inclusions are more likely to affect normal cellular control of the synthesis, the 
folding, trafficking and degradation of proteins (proteostasis), for example, through sequestration of proteins (eg, chaperones) that would otherwise execute normal functions in the cell. Proteostasis is particularly important to tissues with limited capacity for cell renewal; this vulnerability is likely to be aggravated by the reduced expression of adenosine triphosphate-dependent chaperones with age in the brain. ${ }^{9}$ Mutations in the $\tau$ gene are sufficient to cause some NDDs, ${ }^{10}$ providing unequivocal evidence that misfolded $\tau$ in inclusions is toxic. The fact that $\tau$ tangles can misfold in a variety of ways and are associated with a range of different NDD, including AD, progressive supranuclear palsy and frontotemporal dementia, also suggests that different neuronal populations are likely to be vulnerable to specific pathological conformations. The neurons implicated in early $\mathrm{AD}$, including entorhinal cortex and hippocampal CA1 projection neurons, are particularly vulnerable to decreased glucose and oxygen delivery through the vasculature and thus to energy deprivation. ${ }^{11}$ Indeed, mild cognitive dysfunction, which frequently progresses to $\mathrm{AD}$ dementia, correlates with reduced glucose utilisation in the brain as assessed by fluorodeoxyglucose positron emission tomography (FDG-PET). ${ }^{12}$ In addition, synaptic transmission, endoplasmic reticulum stress and calcium homeostasis have been implicated as major targets of diseases in $\mathrm{AD} .{ }^{13} 14$

The connectivity and excitability properties of neuronal subpopulations may play a major role in determining their intrinsic sensitivity to stress. Intrinsic distinctions in the susceptibility of neurons to individual misfolding-prone proteins may account for the broadly similar disease patterns and pathology in sporadic and familial cases of the NDDs. Cascades of mutually reinforcing stress responses may escalate in an age-sensitive manner in affected, stress-sensitive and misfolding-protein-sensitive neurons, causing their dysfunction and death. The accumulation of the toxic protein species may subsequently spread to other less vulnerable cells and increase their stressor load as well. Environmental factors may affect several brain systems through systemic involvement, for example, involving the vasculature, inflammatory responses and the immune system, as well as spreading of toxic protein species. ${ }^{15}$ Selective vulnerability may in part be a consequence of mature or aged neurons being close to different catastrophic cliffs, depending on their function, history of stress exposure and genetic predisposition, that may explain why certain inclusions and aggregates preferentially injure certain types of neurons.

Here, we discuss the selective vulnerability concept in the context of phenotypical variation in AD. In particular, we examine what determines differences in neuronal dysfunction and degeneration, and consequently phenotype, in amnestic AD versus $\mathrm{AD}$ presenting as lvPPA, PCA, CBS or dysexecutive and behavioural variants of $\mathrm{AD}$ that are all characterised by classic $\mathrm{AD}$ plaque and tangle pathology. Focusing on the similarities and differences between these variants may be useful to identify molecular pathways that may underlie selective vulnerability; these may give important clues on pathogenic mechanisms and hopefully also generate new ideas on potential targets for treatment other than the traditional $\tau$-based and $A \beta$-based approaches.

\section{CLINICAL VARIANTS OF ALZHEIMER'S DISEASE}

In terms of neuropathology, $\mathrm{AD}$ is defined as a brain disease with accumulation of $A \beta$ plaques (senile plaques, extracellular deposits of $A \beta$ peptides), neuritic plaques (a subset of senile plaques, defined by the presence of phospho- $\tau(\mathrm{P}-\tau)$ immunoreactivity) and neurofibrillary tangles (intraneuronal fibrils of abnormal $\tau) .{ }^{16}$ During the past decade, it has become increasingly clear that this neuropathology is shared between patients with quite different clinical disease presentations that are all considered to be variants of $\mathrm{AD}$. Late-onset $\mathrm{AD}$ (LOAD, arbitrarily defined as age-at-onset $>65$ years) is by far the most common variant and typically presents with episodic memory deficits. Early-onset AD (EOAD) is markedly less common, and may occur on a monogenic basis which itself may have a variety of different phenotypes at least in part driven by the specific causative mutation. ${ }^{17}$ In patients with apparently sporadic EOAD, compared to patients with LOAD, more impaired attention, language, visuospatial abilities and executive functions are seen. Several focal $\mathrm{AD}$ variants with dominating non-amnestic symptomatology have been described; these are most commonly seen in EOAD. ${ }^{18} 19$ Instead of memory deficiency, these entities are characterised by predominant deficits in language (lvPPA), visuospatial (PCA), motor (CBS) and executive or behavioural functions. Both lvPPA ${ }^{20}$ and $\mathrm{PCA}^{421}$ are most often caused by underlying $\mathrm{AD}$ neuropathology, but may also be caused by other pathologies such as frontotemporal lobar degeneration pathology for lvPPA, ${ }^{20}$ and Lewy body disease and corticobasal degeneration for PCA. ${ }^{4}$ About $25 \%$ of patients with CBS have $\mathrm{AD}$ as the underlying pathology, but the most common cause of CBS is corticobasal degeneration. ${ }^{22}$ Finally, a rare group of patients with $\mathrm{AD}$ have predominantly behavioural/dysexecutive symptoms (sometimes described as a 'frontal variant' of $\mathrm{AD}$ ). These patients most often present with cognitive symptoms and may be differentiated from behavioural variant FTD by a more restricted behavioural profile and co-occurrence of memory dysfunction (in behavioural AD), or minimal behavioural involvement (in dysexecutive AD). ${ }^{2}$

\section{THE ROLE OF AB PATHOLOGY IN DIFFERENT AD VARIANTS}

Accumulation of $A \beta$ pathology is a necessary (but not sufficient) requirement for development of symptomatic $\mathrm{AD}$. This fact has spurred extensive investigations on the regulation of $A \beta$ production, $A \beta$ accumulation and $A \beta$ spread throughout the human brain. $A \beta$ production is related to neuronal activity in cell models, ${ }^{23}$ animal models ${ }^{24}$ and humans. ${ }^{25}$ It is, therefore, interesting that the pattern of $\mathrm{A} \beta$ accumulation largely overlaps with highly connected brain regions (including, but not limited to the 'default-mode network'26 ${ }^{27}$ ). However, the relationship between connectivity and $A \beta$ pathology is complex since $A \beta$ burden may be more prone to develop and/or spread in a prionlike manner ${ }^{28}$ in the well-connected parts of the brain, and also leads to reduced connectivity in the same brain regions. ${ }^{29}$

The regional pattern of $A \beta$ accumulation provides surprisingly little information about AD phenotypes. Converging data from autopsy studies, imaging studies and cerebrospinal fluid studies suggests that variations in $\mathrm{A} \beta$ pathology explain at most only a small amount of the phenotypical variations. ${ }^{30-33}$ Instead, $A \beta$ pathology appears to be diffusely distributed in a relatively similar fashion, without major differences between clinical variants of AD. ${ }^{31} 32{ }^{34}$ However, these studies have been performed on patients with established disease. Given that $A \beta$ pathology is likely to develop several years or decades prior to clinical onset, it remains possible that $A \beta$ pathology starts to develop in different networks in different $\mathrm{AD}$ variants ${ }^{29}$ before converging at a relatively early stage; this could explain why patients with PCA may have slightly increased occipital A $\beta$ accumulation compared to other $\mathrm{AD}$ variants. ${ }^{31}$ In relation to this, one may also consider findings from a recent cerebrospinal fluid (CSF-MRI) study (restricted to patients with AD with pathological levels of CSF A 342$),{ }^{35}$ where-contrary to the authors' hypothesis-there were associations between lower CSF A $\beta 42$ 
(but not CSF $\tau$ ) and syndrome-specific variations of atrophy, which may suggest that a more advanced $A \beta$ pathology is associated with $\mathrm{AD}$ variant-specific patterns of neuronal injury.

\section{NEURONAL INJURY IN DIFFERENT AD VARIANTS}

Neuronal injury in AD variants has been extensively studied in vivo using three different technologies: cross-sectional volume loss or longitudinal atrophy measured by MRI, hypometabolism measured by FDG-PET and $\tau$ concentrations in CSF (using total- $\tau$ and/or phosphorylated $\tau$ ). A consistent finding is that the clinical $\mathrm{AD}$ variants have different distributions of atrophy and hypometabolism which, in contrast to amyloid burden, much more closely mirror the clinical symptoms. ${ }^{31} 3436$ The lack of anatomic specificity is one of the major limitations of fluid biomarkers in the study of the selective vulnerability concept. CSF total- $\tau$ and phosphorylated $\tau$ may be altered to a similar degree in all $\mathrm{AD}$ variants. ${ }^{35}$

Regarding the distribution of neuronal injury, the typical pattern of volume loss in LOAD is involvement of the medial temporal lobes, hippocampus and parietal lobes; while not always apparent on a single patient level, on a group basis there is volume loss in the posterior cingulate, precuneus and other structures involved in the default mode network. This pattern is also seen in many patients with EOAD, but early onset patients often have a more pronounced posterior cortical hypometabolism, ${ }^{34}$ and more pronounced deficits in cholinergic and other neurotransmitter systems. ${ }^{37}$ Atrophy in lvPPA is focused to the language-dominant left hemisphere, ${ }^{20} 39$ while patients with PCA have atrophy focused to the occipital, parietal, and occipitotemporal cortices. ${ }^{4} 21$ Irrespective of the neuropathology, patients with CBS have asymmetric frontoparietal cortical atrophy, with predominant involvement of premotor cortex, insula, and supplementary motor areas. In addition to these regions, patients with $\mathrm{CBS}-\mathrm{AD}$ also have involvement of the temporoparietal lobes, with a relative sparing of hippocampus ${ }^{22}$ (but this atrophy pattern can also be seen in patients without $\mathrm{AD} \mathrm{CBS}^{40}$ ). Patients with the behavioural or the dysexecutive variant of AD have an atrophy pattern similar to typical amnestic $\mathrm{AD}$, with predominant posterior atrophy rather than frontal atrophy, leading to questions on the designation of 'frontal variant AD'. ${ }^{2}$

Despite the differences in neurodegeneration described above, it should be noted that there is a high degree of overlap between $\mathrm{AD}$ variants with hypometabolism in the dorsal default mode network ${ }^{31}$ and atrophy in parietotemporal regions and posterior cingulate. ${ }^{41}$ Furthermore, with disease progression, the patterns of neurodegeneration converge even further between the variants ${ }^{42}$ making it more difficult to detect variant-specific characteristics in advanced patients.

\section{TAU PATHOLOGY IN DIFFERENT AD VARIANTS}

Autopsy studies have found that $\tau$ pathology is more closely related than $A \beta$ pathology to atrophy and clinical symptoms. ${ }^{44}$ The recent development of $\tau$ PET imaging now allows this to be tested in living patients. One recent case report of a patient with PCA showed a strong spatial overlap between $\tau$ pathology visualised by $\tau$ PET imaging using the 18F-AV1451 tracer and hypometabolism visualised by FDG-PET imaging, ${ }^{45}$ findings which have subsequently been replicated in larger case series. ${ }^{35}$ Notably, although CSF P- $\tau$ concentrations correlate with neurofibrillary pathology in $\mathrm{AD},{ }^{46} 47$ the marker does not provide information on the anatomic location of the pathology, making it less useful than $\tau$ PET imaging in the study of selective vulnerability-related issues.

\section{OTHER PATHOLOGIES}

CSF biomarkers to assess plaque and tangle pathology in AD represent an important step forward, particularly when combined with imaging to assess cerebrovascular pathology and (now) cerebral $\mathrm{A} \beta$ and $\tau$. However, at autopsy, very few (if any) patients have just one 'pure' NDD and in particular, comorbidities including TDP-43 and $\alpha$-synuclein (Lewy body) pathologies are highly prevalent. While such pathologies are unlikely to be simply coincidental, our ability to address any contributory effects in vivo is very limited, ${ }^{48-50}$ reflecting that currently available assays only roughly address total levels, showing no or only minor differences between patients and controls ${ }^{51}{ }^{52}$; key pathogenic species do not appear to have been captured by the assays, at least not in any specific manner; and there are as yet no reliable imaging biomarkers for these inclusions. As and when biomarkers with appropriate sensitivity and specificity become available, it will be possible to test the hypothesis that accumulation of TPD-43 and $\alpha$-synuclein in neurons might make them more susceptible to $\tau$-induced or $A \beta$-induced damage, thereby explaining some of the selective vulnerability seen in typical and variant $A D$.

\section{VARIANT-DEPENDENT INVOLVEMENT OF DIFFERENT FUNCTIONAL NETWORKS}

It is notable that the different patterns of neuronal injury observed in $\mathrm{AD}$ variants broadly map onto established functional networks in the brain. Thus EOAD, language predominant $\mathrm{AD}$, and visuospatial predominant $\mathrm{AD}$ typically show hypometabolism broadly focused in brain areas consistent with executive-control, language and high-order visual networks, respectively. ${ }^{53}$ These findings underlie a model that attempts to integrate findings from $\mathrm{A} \beta, \tau$, and injury studies to explain the variability among $\mathrm{AD}$ phenotypes, ${ }^{31}$ postulating that aggregation of $A \beta$ is driven by the total neuronal activity in highly connected cortical hubs (which explains the diffuse and symmetric patterns of amyloid pathology), while $\tau$ pathology develops in specific vulnerable networks and, possibly facilitated by amyloid pathology, spreads transneuronally to closely related networks. As $\tau$-mediated injury patterns more closely correlate both with specific functional networks and neuronal loss, this provides a means of explaining the clinical variability. ${ }^{54}$ If this model is correct, then the different $\mathrm{AD}$ variants arise due to different localisations of $\tau$-related neuronal injury in specific functional networks. The next logical step is to identify factors that predispose specific networks to $\tau$-mediated injury. It would also be important to examine whether pathology starts within the same hub (eg, the posterior cingulate) and then spreads differently within differently connected parts of the network, or starts within the network and then moves towards the hub, laterally spreading throughout the other interconnected hubs. One study of different primary progressive aphasias found that learning disability, but not left-handedness, was increased in patients with lvPPA (but not in semantic or non-fluent variants of PPA, which are not caused by AD), ${ }^{55}$ suggesting that these patients might have had a long-standing dysfunction or differential development in networks relevant for specific aspects of language.

\section{RESILIENCE FACTORS}

A corollary of such hypotheses is that individuals should be identifiable who have connectivity patterns inherently resistant to the initiation or spread of neurodegeneration. This may be an important factor underlying incomplete penetrance of genetic 
mutations. Differential gene expressions across brain regions may be a further substrate ${ }^{56}$ Akin to cancer, stochastic events at both the genetic or protein homeostatic level may generate 'seeds' for the propagation of neurodegeneration. However, most of these seeds may fall on 'unfertile' brain regions in terms of connectivity or cellular molecular resistance mechanisms. The varying prevalence of neurodegenerative disorders might reflect a more generic difference in the accessibility of brain networks. In the case of amyotrophic lateral sclerosis (ALS), the primary motor cortex may be a relatively small target to hit that is difficult to access by spread from an occipital lobe focus, but more easily via frontotemporal pathways inherently linked to human motor functions. ${ }^{57}$ Once breached, however, the largely monosynaptic motor system may be easily overwhelmed, reflected in the notably more rapid progression of ALS compared to AD. ${ }^{58}$

\section{CHEMICAL ASPECTS}

Could basic chemistry add anything to the selective vulnerability concept? Protein aggregation is concentration-dependent and $\mathrm{pH}$-dependent, with higher concentrations and lower $\mathrm{pH}$ increasing the risk of aggregation. ${ }^{59}$ Increased production or defective clearance of aggregation-prone proteins intracellularly (eg, via autophagy) or extracellularly (eg, via perivascular drainage of $A \beta$ ) may initiate the process. ${ }^{60}$ However, there is no direct link between protein aggregation and toxicity. One intriguing and often overlooked aspect in this context is chemical protein ageing (non-enzymatic post-translational modifications that occur over time, particularly in water-deprived milieus, such as inclusions and aggregates and include methylation, deamidation, $\mathrm{N}$-terminal racemisation and truncation ${ }^{61}$ ), which may increase differential toxicity of the aggregated protein. One hypothesis, supported by data from the prion field ${ }^{62}$ as well as by results showing that brain-incubated $A \beta$ is more potent than synthetic $A \beta$ aggregates in regards to its infectivity and toxicity, ${ }^{63}$ is that $A \beta$ build-up and toxicity may occur in two phases: the first involving build-up of a non-toxic, virtually inert $A \beta$ reservoir, and the second involving time-dependent chemical modification and destabilisation of plaque-incubated $A \beta$, which eventually results in induction of microglial activation, tangle pathology and neurodegeneration (ie, gain of toxicity). If so, what factors govern this process are currently unknown, but the concept is supported by recent data showing more $\mathrm{N}$-terminal truncations and pyroglutamate modifications (both of which increase over time as a consequence of protein ageing) in apparently toxic $A \beta$ aggregates isolated from $\mathrm{AD}$ brains, compared with those (probably nontoxic plaques) isolated from cognitively normal individuals who fulfilled criteria for pathological ageing at autopsy (AD-like brain changes without cognitive dysfunction) ${ }^{64}$ Similar experiments performed on $A \beta$ pathology in different brain regions primarily affected in different $\mathrm{AD}$ presentations, for example, the hippocampus in typical AD and occipital cortex in PCA might therefore provide a means of testing this hypothesis. If gain of toxicity is simply a function of time, the localisation of the first $A \beta$ seeds could determine where the most severe neurodegeneration and symptoms eventually appear; degenerated areas should express more aged $A \beta$ fragment profiles than areas with plaque pathology surrounded by apparently healthy brain tissue. Such a scenario might provide important insights into selective vulnerability within a given network.

\section{CONCLUDING REMARKS}

The selective neuronal vulnerability concept tries to explain why only some neurons succumb in the presence of neuropathology, and why certain NDDs devastate certain neuronal networks while leaving others intact. Here, we have discussed this with special emphasis on clinical variants of $\mathrm{AD}$. The aim has been to gather neuroimaging and neurochemical data that may provide useful leads to toxicity mechanisms in the brain in these disorders. It is clear that there is as yet unexplained dissociation between the topographical distribution of key pathologies, that is, $\mathrm{A} \beta$ and $\tau$ in the case of $\mathrm{AD}$, and that certain neuronal networks may be more vulnerable than others. The precise cascade of pathological events underlying $\mathrm{AD}$ is being elucidated only recently; to date there is relatively little data in the literature on the genetic, physiological or biochemical factors influencing neuronal vulnerability or for that matter, resilience factors. While cross-disease comparisons are required to determine the factors influencing why specific proteinopathies affect certain networks, there is much to be learnt from studies of phenotypically different presentations of the same disease. We propose that large, consortia-based studies of deeply phenotyped patients with amnestic and variant forms of $\mathrm{AD}$ will be one way to explore selective vulnerability mechanisms in greater detail. There is value in comparing prognostic extremes within the same clinical phenotype, as well as unaffected older populations (the 'wellderly'), especially asymptomatic carriers of typically penetrant mutations. Determining the factors influencing neuronal vulnerability or resilience may in due course provide insights into novel treatment paradigms.

Contributors $\mathrm{NM}$ and $\mathrm{HZ}$ drafted the paper. All authors have made substantial contributions to the conception and design of the work, the acquisition, analysis and interpretation of data for the work. All authors took part in revising it critically for important intellectual content. All authors approved the final version. All authors are in agreement to be accountable for all aspects of the work by ensuring that questions related to the accuracy or integrity of any part of the work are appropriately investigated and resolved.

Funding Work in the authors' laboratories is supported by the Swedish Research Council (NM and HZ), the Wolfson Foundation (JS, JH and HZ), the NIHR UCL/H Biomedical Research Centre and Queen Square Dementia BRU (JMS), Alzheimer's Research UK (JMS), the Medical Research Centre (JMS), the Medical Research Council (MRT), Motor Neurone Disease Association Lady Edith Wolfson Senior Clinical Fellowship (MRT), the Knut and Alice Wallenberg Foundation (HZ), Frimurarestiftelsen (HZ), and Swedish State Support for Clinical Research (HZ). The Dementia Research Centre (JMS) is an ARUK coordinating Centre.

Competing interests None declared.

Provenance and peer review Commissioned; externally peer reviewed.

\section{REFERENCES}

1 Warren JD, Fletcher PD, Golden HL. The paradox of syndromic diversity in Alzheimer disease. Nat Rev Neurol 2012;8:451-64

2 Ossenkoppele R, Pijnenburg YA, Perry DC, et al. The behavioural/dysexecutive variant of Alzheimer's disease: clinical, neuroimaging and pathological features. Brain 2015;138(Pt 9):2732-49.

3 Ahmed S, de Jager CA, Haigh AM, et al. Logopenic aphasia in Alzheimers disease: clinical variant or clinical feature? J Neurol Neurosurg Psychiatry 2012;83:1056-62.

4 Crutch SJ, Lehmann M, Schott JM, et al. Posterior cortical atrophy. Lancet Neurol 2012;11:170-8

5 Grijalvo-Perez AM, Litvan I. Corticobasal degeneration. Semin Neurol 2014;34:160-73

6 Baron JC, Yamauchi H, Fujioka M, et al. Selective neuronal loss in ischemic stroke and cerebrovascular disease. J Cereb Blood Flow Metab 2014;34:2-18.

7 Fehm HL, Kern W, Peters A. The selfish brain: competition for energy resources. Prog Brain Res 2006;153:129-40.

8 Polymenidou M, Cleveland DW. Prion-like spread of protein aggregates in neurodegeneration. J Exp Med 2012;209:889-93.

9 Yang $S$, Huang S, Gaertig MA, et al. Age-dependent decrease in chaperone activity impairs MANF expression, leading to Purkinje cell degeneration in inducible SCA17 mice. Neuron 2014;81:349-65.

10 McCarthy A, Lonergan R, Olszewska DA, et al. Closing the tau loop: the missing tau mutation. Brain 2015:138(Pt 10):3100-9.

11 Hof PR, Morrison JH. The aging brain: morphomolecular senescence of cortical circuits. Trends Neurosci 2004;27:607-13. 
12 Reiman EM. Fluorodeoxyglucose positron emission tomography: emerging roles in the evaluation of putative Alzheimer's disease-modifying treatments. Neurobiol Aging 2011;32(Suppl 1):S44-7.

13 Bezprozvanny I, Mattson MP. Neuronal calcium mishandling and the pathogenesis of Alzheimer's disease. Trends Neurosci 2008;31:454-63.

14 Wasling P, Daborg J, Riebe I, et al. Synaptic retrogenesis and amyloid-beta in Alzheimer's disease. J Alzheimers Dis 2009;16:1-14.

15 Saxena S, Caroni P. Selective neuronal vulnerability in neurodegenerative diseases: from stressor thresholds to degeneration. Neuron 2011;71:35-48.

16 Hyman BT, Phelps CH, Beach TG, et al. National Institute on Aging-Alzheimer's Association guidelines for the neuropathologic assessment of Alzheimer's disease. Alzheimers Dement 2012;8:1-13.

17 Ryan NS, Rossor MN. Defining and describing the pre-dementia stages of familial Alzheimer's disease. Alzheimers Res Ther 2011;3:29.

18 Schott JM, Warren JD. Alzheimer's disease: mimics and chameleons. Pract Neurol 2012;12:358-66.

19 Rossor MN, Fox NC, Mummery CJ, et al. The diagnosis of young-onset dementia. Lancet Neurol 2010;9:793-806.

20 Mesulam M, Wicklund A, Johnson N, et al. Alzheimer and frontotemporal pathology in subsets of primary progressive aphasia. Ann Neurol 2008;63:709-19.

21 Benson DF, Davis RJ, Snyder BD. Posterior cortical atrophy. Arch Neurol 1988;45:789-93.

22 Hassan A, Whitwell JL, Josephs KA. The corticobasal syndrome-Alzheimer's disease conundrum. Expert Rev Neurother 2011;11:1569-78.

23 Kamenetz F, Tomita T, Hsieh H, et al. APP processing and synaptic function. Neuron 2003;37:925-37

24 Bero AW, Yan P, Roh JH, et al. Neuronal activity regulates the regional vulnerability to amyloid-beta deposition. Nat Neurosci 2011;14:750-6.

25 Brody DL, Magnoni S, Schwetye KE, et al. Amyloid-beta dynamics correlate with neurological status in the injured human brain. Science 2008;321:1221-4.

26 Buckner RL, Snyder AZ, Shannon BJ, et al. Molecular, structural, and functiona characterization of Alzheimer's disease: evidence for a relationship between default activity, amyloid, and memory. J Neurosci 2005;25:7709-17.

27 Jagust WJ, Mormino EC. Lifespan brain activity, beta-amyloid, and Alzheimer's disease. Trends Cogn Sci (Regul Ed) 2011;15:520-6.

28 Walker LC, Jucker M. Neurodegenerative diseases: expanding the prion concept. Annu Rev Neurosci 2015;38:87-103.

29 Myers N, Pasquini L, Göttler J, et al. Within-patient correspondence of amyloid-beta and intrinsic network connectivity in Alzheimer's disease. Brain 2014;137(Pt 7):2052-64.

30 de Souza LC, Corlier F, Habert MO, et al. Similar amyloid-beta burden in posterior cortical atrophy and Alzheimer's disease. Brain 2011;134(Pt 7):2036-43.

31 Lehmann M, Ghosh PM, Madison C, et al. Diverging patterns of amyloid deposition and hypometabolism in clinical variants of probable Alzheimer's disease. Brain 2013;136(Pt 3):844-58

32 Rosenbloom MH, Alkalay A, Agarwal N, et al. Distinct clinical and metabolic deficits in PCA and AD are not related to amyloid distribution. Neurology 2011;76:1789-96.

33 Teng E, Yamasaki TR, Tran M, et al. Cerebrospinal fluid biomarkers in clinical subtypes of early-onset Alzheimer's disease. Dement Geriatr Cogn Disord 2014:37:307-14

34 Rabinovici GD, Furst AJ, Alkalay A, et al. Increased metabolic vulnerability in earlyonset Alzheimer's disease is not related to amyloid burden. Brain 2010;133 (Pt 2):512-28.

35 Ossenkoppele R, Mattsson N, Teunissen CE, et al. Cerebrospinal fluid biomarkers and cerebral atrophy in distinct clinical variants of probable Alzheimer's disease. Neurobiol Aging 2015;36:2340-7.

36 Migliaccio R, Agosta F, Rascovsky K, et al. Clinical syndromes associated with posterior atrophy: early age at onset AD spectrum. Neurology 2009;73:1571-8.

37 Bird TD, Stranahan S, Sumi SM, et al. Alzheimer's disease: choline acetyltransferase activity in brain tissue from clinical and pathological subgroups. Ann Neurol 1983:14:284-93.

38 Rossor MN, Iversen LL, Reynolds GP, et al. Neurochemical characteristics of early and late onset types of Alzheimer's disease. Br Med J (Clin Res Ed) 1984;288:961-4.

39 Gorno-Tempini ML, Brambati SM, Ginex V, et al. The logopenic/phonological variant of primary progressive aphasia. Neurology 2008;71:1227-34.
40 Sha SJ, Ghosh PM, Lee SE, et al. Predicting amyloid status in corticobasal syndrome using modified clinical criteria, magnetic resonance imaging and fluorodeoxyglucose positron emission tomography. Alzheimers Res Ther 2015;7:8.

41 Whitwell JL, Jack CR Jr, Przybelski SA, et al. Temporoparietal atrophy: a marker of $A D$ pathology independent of clinical diagnosis. Neurobiol Aging 2011;32:1531-41.

42 Lehmann M, Barnes J, Ridgway GR, et al. Global gray matter changes in posterior cortical atrophy: a serial imaging study. Alzheimers Dement 2012;8:502-12.

43 Migliaccio R, Agosta F, Possin KL, et al. Mapping the progression of atrophy in early- and late-onset Alzheimer's disease. J Alzheimers Dis 2015;46:351-64.

44 Mitchell TW, Mufson EJ, Schneider JA, et al. Parahippocampal tau pathology in healthy aging, mild cognitive impairment, and early Alzheimer's disease. Ann Neurol 2002;51:182-9.

45 Ossenkoppele R, Schonhaut DR, Baker SL, et al. Tau, amyloid, and hypometabolism in a patient with posterior cortical atrophy. Ann Neurol 2015;77:338-42.

46 Buerger K, Ewers M, Pirttilä T, et al. CSF phosphorylated tau protein correlates with neocortical neurofibrillary pathology in Alzheimer's disease. Brain 2006;129(Pt 11):3035-41.

47 Seppälä TT, Nerg 0, Koivisto AM, et al. CSF biomarkers for Alzheimer disease correlate with cortical brain biopsy findings. Neurology 2012;78:1568-75.

48 Nag S, Yu L, Capuano AW, et al. Hippocampal sclerosis and TDP-43 pathology in aging and Alzheimer's disease. Ann Neurol 2015;77:942-52.

49 Schneider JA, Arvanitakis Z, Yu L, et al. Cognitive impairment, decline and fluctuations in older community-dwelling subjects with Lewy bodies. Brain 2012;135(Pt 10):3005-14.

50 Josephs KA, Whitwell JL, Weigand SD, et al. TDP-43 is a key player in the clinical features associated with Alzheimer's disease. Acta Neuropathol 2014;127:811-24.

51 Feneberg E, Steinacker $\mathrm{P}$, Lehnert $\mathrm{S}$, et al. Limited role of free TDP-43 as a diagnostic tool in neurodegenerative diseases. Amyotroph Lateral Scler Frontotemporal Degener 2014;15:351-6.

52 Magdalinou N, Lees AJ, Zetterberg H. Cerebrospinal fluid biomarkers in parkinsonian conditions: an update and future directions. J Neurol Neurosurg Psychiatry 2014;85:1065-75.

53 Laforce R Jr, Tosun D, Ghosh P, et al. Parallel ICA of FDG-PET and PiB-PET in three conditions with underlying Alzheimer's pathology. Neuroimage Clin 2014:4:508-16.

54 Lehmann M, Madison CM, Ghosh PM, et al. Intrinsic connectivity networks in healthy subjects explain clinical variability in Alzheimer's disease. Proc Natl Acad Sci USA 2013;110:11606-11.

55 Miller ZA, Mandelli ML, Rankin KP, et al. Handedness and language learning disability differentially distribute in progressive aphasia variants. Brain 2013;136(Pt 11):3461-73.

56 Yokoyama JS, Sirkis DW, Miller BL. C9ORF72 hexanucleotide repeats in behavioral and motor neuron disease: clinical heterogeneity and pathological diversity. Am J Neurodegener Dis 2014;3:1-18.

57 Eisen A, Turner MR, Lemon R. Tools and talk: an evolutionary perspective on the functional deficits associated with amyotrophic lateral sclerosis. Muscle Nerve 2014;49:469-77

58 Eisen A, Turner MR. Does variation in neurodegenerative disease susceptibility and phenotype reflect cerebral differences at the network level? Amyotroph Lateral Scler Frontotemporal Degener 2013;14(7-8):487-93.

59 Buell AK, Dobson CM, Knowles TP. The physical chemistry of the amyloid phenomenon: thermodynamics and kinetics of filamentous protein aggregation. Essays Biochem 2014;56:11-39.

60 Tarasoff-Conway JM, Carare RO, Osorio RS, et al. Clearance systems in the brain-implications for Alzheimer disease. Nat Rev Neurol 2015;11:457-70.

61 Lyons B, Kwan AH, Jamie J, et al. Age-dependent modification of proteins: N-terminal racemization. FEBS J 2013;280:1980-90.

62 Sandberg MK, Al-Doujaily $H$, Sharps B, et al. Prion propagation and toxicity in vivo occur in two distinct mechanistic phases. Nature 2011;470:540-2.

63 Eisenberg D, Jucker M. The amyloid state of proteins in human diseases. Cell 2012;148:1188-203.

64 Portelius E, Lashley T, Westerlund A, et al. Brain amyloid-beta fragment signatures in pathological ageing and Alzheimer's disease by hybrid immunoprecipitation mass spectrometry. Neurodegener Dis 2015;15:50-7. 